Rev. Saúde públ., S. Paulo, 19:556-65, 1985

\title{
CONCEPÇÃO E ORGANIZAÇÃO DE UM CENTRO DE CONTROLE DE INTOXICAÇÓES*
}

Ricardo Baroud**

BAROUD, R. Concepção e organização de um centro de controle de intoxicaçōes. Rev. Saúde públ., S. Paulo, $19: 556-65,1985$.

RESUMO: Objetivou-se contribuir para uma melhor compreensão da importância dos Centros de Controle de Intoxicaçōes (ou Centros Anti-Veneno), através do relato de sua concepção e organização. Ressaltou-se a necessidade destes centros atuarem integrados a instituiçōes governamentais, a entidades privadas e à comunidade, e a importância dos setores de epidemiologia e prevenção, de análises toxicológicas e de pesquisa científica nestes centros. Enfatizou-se a necessidade de sua intervenção na área de promoção e proteção à saúde, através do estabelecimento de linhas de ação principalmente a nível educacional, de saúde mental e de capacitação profissional. Foram sugeridas algumas providências que concorrerão para maior esclarecimento e, consequentemente, para a diminuição da incidência de intoxicaçōes, revertendo em economia de recursos financeiros que são necessários ao atendimento integral de pacientes intoxicados.

UNITERMOS: Centros de Controle de Envenenamento. Envenenamento, prevenção e controle.

\section{INTRODUÇĀO}

Este trabalho objetiva apresentar uma contribuição quanto à concepção e organização de um Centro de Controle de Intoxicações $(\mathrm{CCI})$, denominado, também, Centro Anti-Veneno. Visa, ainda, ressaltar a importância de ser a ação destes centros integrada a de instituições governamentais, entidades privadas e comunidade. O critério proposto neste trabalho como parâmetro para a organização de um CCI pode ser admitido como ideal e baseia-se naquele estabelecido e recomendado durante a conferência do "Regional Bureau in Europe of World Health Organization", Istambul, 1965 (Govaerts ${ }^{13}$ ), tendo sido sua importância enfatizada, também, por Sunshine e col. ${ }^{32}$ (1966). Espera-se desta forma, contribuir para o esclarecimento de algumas das questões existentes em torno deste tema.

\section{HISTORICO DO SURGIMENTO DAS ENTIDADES LIGADAS À INTOXICAÇÖES}

Será exposto um histórico sucinto sobre o surgimento das entidades ligadas às intoxicações nos diversos continentes, com a finalidade de dimensionar a real importância e abrangência das mesmas frente à área de saúde pública, através do conhecimento de suas ações.

\section{Continente Americano}

Inaugurou-se, em novembro de 1953 , na cidade de Chicago (EUA), o primeiro centro ligado diretamente à problemática das intoxicações exógenas, sob a denominação de "Poison Control Center" . Esta iniciativa deveu-se aos esforços do "Illinois Chapter of American Academy of Pediatrics" e de outras entidades ligadas à área. Este centro tinha por ob-

\footnotetext{
- Trabalho apresentado no III Congresso Brasileiro de Toxicologia e I Congresso Brasileiro de Acidentes e Intoxicação na Infância, Belo Horizonte, MG, agosto de 1983.

- Do Centro de Controle de Intoxicaçōes da Prefeitura do Município de São Paulo - Av. Francisco de Paula Quintanilha Ribeiro, 860 - 04330 - São Paulo, SP - Brasil.
} 
BAROUD, R. Concepção e organização de um centro de controle de intoxicaçōes. Rev. Saúde públ., S. Paulo, 19:556-65, 1985.

jetivos fornecer, aos profissionais da área, informações e conduta terapêutica acerca das intoxicações exógenas, além de desenvolver um programa de prevenção de acidentes toxicológicos. Outras cidades dos EUA seguiram o exemplo de Chicago e organizaram centros com metas similares. Em 1956, sentindo a necessidade de um organismo nacional que interligasse todos os centros já existentes, representantes dos mesmos e de entidades atuantes na área de saúde pública criaram o "National Clearinghouse for Poison Control Centers" ". A função deste organismo nacional era a de recolher, catalogar e fornecer informações clínicas e toxicológicas, sistematizadas, aos centros já organizados, além de coordenar os programas de prevenção de acidentes toxicológicos. No início de 1958 , os dezessete centros autônomos já estabelecidos fundaram o "American Association of Poison Control Centers", que deveria propiciar o inter-relacionamento, aprimorar a eficiência e, ainda, planejar o desenvolvimento daqueles centros a serem implementados ${ }^{6}$. A partir deste momento, o número de centros cresceu rapidamente nos EUA. Em 1961, estavam em operação 462 cetnros $^{31}$ e, em 1978, este número se elevava a $661^{27}$. No Canadá, a partir da criação do primeiro centro, em 1957, houve rápida proliferação destes organismos, existindo $222^{31}$ centros em $1963 \mathrm{e}$, atualmente, cerca de 300 . Estes centros estão, primariamente, sob a responsabilidade dos governos locais, sendo coordenados por uma agência localizada em Ottawa, subordinada ao "Food and Drug Directorate". Por sua vez, o México estabeleceu, em 1966, na cidade do México, o Centro de Intoxicados, no "Hospital de Pediatría del Centro Médico Nacional", ligado ao "Instituto Mexicano del Seguro Social"'22. Na América do Sul, a Argentina já contava, desde 1962, com o "Centro de Intoxicaciones" localizado no "Hospital de Niños", na Província de Buenos Aires ${ }^{31}$. Além deste centro, a Argentina possui, na atualidade, outros dez centros ligados às intoxicações exógenas. O Uruguai, em dezembro de 1975, inaugurou o "Centro de Informacion y Asesoriamiento Toxicologico", localizado em Montedivéu ${ }^{5}$. Este centro encontra-se subordinado administrativamente à "Facultad de Medicina de la Universidad de la Republica". Outros países, seja da América Central, seja da América do Sul, também se propuseram a criar centros similares como, por exemplo, Costa Rica, Venezuela e Colômbia $^{3}$.

\section{Continente Europeu}

$\mathrm{Na}$ Europa, a data de fundação do primeiro centro não está estabelecida rigorosamente. Sabe-se que existia, em 1949, um serviço de toxicologia clínica em Budapeste (Bulgária) e, à mesma época, um serviço de informações, menor, em Leeds (Inglaterra) ${ }^{13}$. No mesmo ano, uma organização farmacêutica holandesa implantou um arquivo contendo informações sobre produtos comerciais, com o objetivo de servir como fonte de referência para farmacêuticos que atuassem na área de envenenamentos ${ }^{12}$. Ainda no início da década de 50, o "Bispebjerg Hospitalet", em Copenhague (Dinamarca), notabilizou-se pela eficiência no tratamento de intoxicações barbitúricas agudas $^{12}$. Com o estabelecimento de uma unidade de cuidados intensivos voltada às intoxicações, no "Hôpital Fernand Widal", em Paris (França), em 1959, e do "National Poison Information Service" na Grã Bretanha, em 1963, foram dados importantes passos para o desenvolvimento destes centros na Europa ${ }^{13}$. Assim, foi criado, já em 1964, o "European Association of Poison Control Centers" e, em 1970, 22 países europeus possuiam programas de controle de intoxicações, sendo que a maioria apresentava um serviço permanente de informação e tratamento médico ${ }^{13}$. 
BAROUD, R. Concepção e organização de um centro de controle de intoxicações. Rev. Saúde públ., S. Paulo, 19:556-65, 1985.

\section{Outros Continentes}

No continente africano, pode-se destacar o Marrocos, que já conta com três centros ligados às intoxicações, além da Tunísia, Egito, Argélia e República SulAfricana $^{3}$. No continente asiático, o Japão conta, atualmente, com três centros de controle de intoxicações e outros dois centros assessores ligados indiretamente às intoxicações. Existem, ainda, centros similares na República Popular da China, Síria, Israel, Iraque, Irã, República Socialista do Vietnã e nas Filipinas ${ }^{3}$. E, finalmente, na Oceania, destaca-se a Austrália com oito centros de informação toxicológica, seguida pela Nova Zelândia e Guam ${ }^{3}$.

\section{Brasil}

No Brasil, o primeiro destes centros surgiu na cidade de São Paulo (SP), em 1971, inaugurado pela Prefeitura do Município de São Paulo sob a denominação de Centro de Controle de Intoxicações ${ }^{8}$. Suas atribuições iniciais eram as de assistir às intoxicações exógenas pediátricas, além de atuar nos campos da prevenção, da pesquisa e da informação toxicológica. A partir de maio de 1980 , seus serviços médicos foram estendidos para o tratamento de adultos intoxicados. Em 1976, a Secretaria da Saúde e do Meio Ambiente do Estado do Rio Grande do Sul criou o Centro de Informação Toxicológica com a finalidade de elaborar um fichário de informações toxicológicas sobre substâncias químicas potencial e efetivamente tóxicas, coletar dados epidemiológicos e fomentar programas educativos. Em fevereiro de 1980, o Ministério da Saúde estabeleceu o Sistema $\mathrm{Na}$ cional de Informações Tóxico-Farmacológicas (SNITF), coordenado e administrado pela Fundação Oswaldo Cruz. O SNITF tem a finalidade de criar, apoiar e integrar os centros regionais. Deve, ainda, desenvolver programas de educa- ção e prevenção de acidentes toxicológicos, além de propiciar a reciclagem e treinamento de profissionais da área de saúde. Atualmente, já existem outros centros regionais de informação estabelecidos em Curitiba (PR), Campo Grande (MS), Rio de Janeiro (RJ), Natal (RN), Florianópolis (SC), João Pessoa (PB), Belo Horizonte (MG) e Brasília (DF), além do Centro de Informações Anti-Veneno em Salvador (BA). No Estado de São Paulo, a exemplo do Centro de Controle de Intoxicações do $\mathrm{Mu}$ nicípio de São Paulo, estabeleceram-se outros centros nas cidades de Campinas, Ribeirão Preto, Botucatu, Sorocaba e Taubaté. Estes centros são orientados pela Coordenadoria Regional dos Centros de Informação Toxicológica situada na Capital e integrada ao SNITF.

\section{CLASSIFICAÇĀO DOS CENTROS \\ LIGADOS ÀS INTOXICAÇÓES SEGUNDO OS TIPOS DE SERVIÇOS OFERECIDOS}

Miller e Johnson (1966) ${ }^{20}$ propuseram uma forma de classificar os centros ligados às intoxicações exógenas que é útil para a compreensão da abrangência da atuação dos diferentes tipos de entidades existentes em vários países. Esta classificação baseia-se nos tipos de serviços oferecidos, e de acordo com a metodologia proposta por aqueles autores, tem-se: (i) centro regional de informação toxicológica, (ii) centro de informação toxicológica, (iii) centro de controle de intoxicações e, (iv) centro de intoxicações.

\section{Centros Regionais de Informação Toxicológica (CRIT)}

Caracterizam-se por atuar como fontes centrais de informações sobre substâncias tóxicas, fornecendo-as eficiente e acuradamente a todos os outros tipos de centros, ininterruptamente. Devem estar localizados, preferencialmente, em instituições de ensino e pesquisa que pos- 
BAROUD, R. Concepção e organização de um centro de controle de intoxicações. Rev. Saúde públ., S. Paulo, 19:556-65, 1985.

suem serviços de urgência médica. A pesquisa, coleta e armazenamento de dados, bem como o sistema de informações, devem ser os mais eficientes possíveis utilizando, de preferência, um processo computorizado.

\section{Centros de Informação Toxicológica (CIT)}

São entidades que têm como única finalidade oferecer serviços de informações. Em sua maioria, nem se localizam em hospitais nem oferecem serviços médicos. Após o desenvolvimento integral dos outros tipos de centros, estes, segundo aqueles autores ${ }^{20}$, deixarão de ter finalidade.

\section{Centros de Controle de Intoxicações (CCI)}

Localizam-se em hospitais que possuem serviços de urgência médica, contando com equipe multidisciplinar. Possuem um serviço de informações especializadas, porém menos complexo que o do CRIT.

\section{Centros de Intoxicações (CI)}

Caracterizam-se por ter, como função principal, a de proporcionar um serviço médico adequado. Eles devem, necessariamente, estar localizados em hospitais. Possuem apenas fontes de referência simples, utilizando os CRITs (ou os outros centros) quando há necessidade de informações mais complexas. Os profissionais que atuam neste centro fazem parte do corpo de funcionários do hospital a que ele está ligado. São, claramente, centros bem menos especializados que os anteriores.

A decisão sobre qual destes Centros é o mais adequado para implantação, numa região urbana ou rural, deve ser tomada em função da infra-estrutura local, quer de atenção à saúde, quer dos meios de comunicação, bem como das características e necessidade da comunidade que, por sua vez, serão determinantes da infra-estrutura do próprio centro e do nível de especialização de seu corpo de funcionários. Para a decisão se faz necessário, evidentemente, um estudo sócioeconômico-cultural preliminar da comunidade.

\section{CONCEPÇÃO E ORGANIZAÇÃO DE UM CENTRO DE CONTROLE DE INTOXICAÇOES}

Pode-se, enfim, definir os CCIs segundo sua atuação. São entidades que visam atuar em todas as áreas que dizem respeito às intoxicações, isto é, no controle e na pesquisa, além de fornecer informações a profissionais e ao público em geral. Visam, também, diagnosticar e tratar os pacientes intoxicados, coletar informações e avaliar, permanentemente, os riscos de intoxicações e os dados epidemiológicos. Estes centros devem estar vinculados a instituições de assistência médica que possuam estrutura e infraestrutura adequadas ao tratamento especializado e de urgência funcionando vinte quatro horas por dia, os sete dias da semana.

Um CCI pode ser subdividido em cinco setores, que se encontram intrinsecamente integrados. Sendo assim, esta subdivisão se refere, principalmente, à melhor compreensão de sua organização e funcionamento. Estes setores são os de (i) informação, (ii) atendimento médico, (iii) análises toxicológicas, (iv) epidemiologia e prevenção e, (v) pesquisa científica.

\section{Setor de Informação}

Tem por objetivo localizar, catalogar, armazenar e fornecer informaçóes acerca de substâncias potencial e efetivamente tóxicas. Estas informações referem-se, principalmente, à: (a) propriedades físico-químicas, mecanismo de ação e toxicidade de substâncias químicas; (b) composição, manipulação e uso adequados 
BAROUD, R. Concepção e organização de um centro de controle de intoxicações. Rev. Saúde públ., S. Paulo, 19:556-65, 1985.

de produtos industrializados e, (c) primeiros-socorros e conduta terapêutica adequada aos casos de intoxicação por produtos de uso industrial, domissanitários, drogas, praguicidas, cosméticos, agentes tóxicos de origem vegetal, toxinas animais e/ou outras substâncias biológica e farmacologicamente ativas. Estas informações são obtidas através de fontes de referência, órgãos oficiais, fabricantes industriais, entidades científicas e dados clínico-laboratoriais obtidos ao longo do tempo pelos centros nacionais e internacionais. Profissionais especializados do CCI forneceriam estes dados aos profissionais de saúde, a entidades públicas e privadas e ao público em geral.

\section{Setor de Atendimento Médico}

Este setor tem por finalidade proporcionar atendimento médico completo aos pacientes intoxicados. Para tanto, a instituição de assistência médica à qual o centro estiver vinculado deve possuir os serviços de: (a) urgências médicas, (b) atendimento de retaguarda com unidade de terapia intensiva equipada adequadamente $e$ arsenal terapêutico específico (agentes antagonistas) para as diferentes síndromes tóxicas $e,(c)$ atendimento psicossocial. Deve atuar neste setor uma equipe multidisciplinar composta de médicos e para-médicos com profundo conhecimento em toxicologia clínica, trabalhando em regime de plantão contínuo, além de profissionais atuantes na área psicossocial (psicólogos, psiquiatras e assistentes sociais).

\section{Setor de Análises Toxicológicas}

Visa, basicamente, o desenvolvimento, a padronização e a implantação de técnicas analíticas para fins de identificação e, se necessário, quantificação das possíveis substâncias e/ou classes químicas presentes no material biológico, quando as mesmas estão envolvidas nu- ma intoxicação exógena. Consequientemente, este setor deve auxiliar no diagnóstico, na conduta terapêutica e/ou no prognóstico destas intoxicações, além de executar o controle dos níveis sanguíneos de drogas com a finalidade de acompanhar a eficácia do tratamento clínico. Pode, ainda, quando se fizer necessário, realizar as análises clínicas complementares ao diagnóstico e tratamento das síndromes tóxicas.

As técnicas analíticas de urgência devem, sempre que possível e, levando-se em consideração a significância clínica dos resultados, possuir as seguintes características: rapidez, simplicidade, sensibilidade e especificidade. Os métodos de determinação qualitativa são empregados na triagem e identificação de grupos e/ou substâncias químicas. Os mais simples, e por isso mais utilizados, são os testes colorimétricos imediatos e as cromatografias em papel e em camada delgada. Os métodos de determinação quantitativa são empregados na confirmação dos métodos de triagem, na quantificação de substâncias conhecidas, ou ainda, no controle dos níveis sanguíneos de drogas. Em função do equipamento disponível, das condições financeiras e do espaço físico existente, pode-se utilizar os seguintes métodos de quantificação: colorimetria, espectrofotometria de infra-vermelho, visível e ultra-violeta, espectrofluorimetria, cromatografia de gás e de líquido, espectroscopia de absorção atômica e imunoensaios.

A realização de um controle de qualidade das análises executadas, quer internamente, quer entre laboratórios do mesmo âmbito, é uma prática que se faz necessária. Em alguns países, como por exemplo nos EUA, este controle de qualidade é exigido por lei, possuindo regulamentação específica para os laboratórios ${ }^{7}$.

Este setor deve atender não só ao centro mas a qualquer outra entidade que 
BAROUD, R. Concepção e organização de um centro de controle de intoxicações. Rev. Saúde públ., S. Paulo, 19:556-65, 1985.

necessite de seus serviços, fornecendo seus resultados o mais breve possível através de telefone ou qualquer outro meio de comunicação disponível. Deve, ainda, ser coordenado por profissionais que possuam sólidos conhecimentos teóricos e analíticos em toxicologia e bioquímica sendo que, atualmente, o farmacêutico-bioquímico (modalidade Análises Clínicas e Toxicológicas) vem exercendo esta atividade em nosso país.

\section{Setor de Epidemiologia e Prevenção}

Neste setor são efetuados estudos epidemiológicos das diversas síndromes tóxicas com a finalidade de revelar os fatores etiológicos envolvidos e, consequientemente, permitir o desenvolvimento de programas destinados à modificação, ao controle ou à prevenção das situações que envolvam riscos toxicológicos. Além disto, estes estudos também tem por objetivos direcionar o desenvolvimento dos CCIs e fornecer uma metodologia de avaliação da eficiência destes centros. A pesquisa epidemiológica das intoxicações compreende uma fase analítica de registro de todos os fatores que atuam no sentido de favorecer, desencadear, acompanhar ou agravar uma intoxicação e uma fase de exploração dos resultados, através de uma análise estatística destes dados, a fim de que se possa planejar linhas de ação preventiva e curativa. Deve-se utilizar, na medida do possível, técnicas de informática na etapa de processamento e análise de dados. Para que se alcancem os objetivos propostos, a equipe multiprofissional do centro deve desenvolver uma estreita colaboração com instituições voltadas para a promoção e proteção da saúde e com segmentos da comunidade.

\section{Setor de Pesquisa Científica}

As metas principais deste setor são: (a) auxiliar as pesquisas básicas através de ensaios que permitam estabelecer com exatidão a toxicocinética, a toxicodinâmica e a síndrome tóxica; (b) desenvolver novos métodos analíticos e terapêuticos, para que se possa obter uma maior eficácia no diagnóstico, na conduta terapêutica e no prognóstico destas síndromes e, (c) realizar quaisquer outras pesquisas complementares que possam contribuir para o desenvolvimento da toxicologia em nosso meio. As informações obtidas através do estabelecimento destas metas e das observações clínico-laboratoriais resultantes do atendimento a pacientes intoxicados serão catalogadas e armazenadas no Setor de Informação Toxicológica, redundando num aumento de seu potencial de ação. Em vista disso, o centro deve possuir um laboratório de toxicologia dotado da infra-estrutura básica para o desenvolvimento de estudos clínico-tóxico-farmacológicos em colaboração com instituições de pesquisa e, ainda, sua equipe deve integrar-se aos grupos de pesquisa multidisciplinar existentes na área.

Os setores de Informação, Atendimento Médico e de Análises Toxicológicas, por se tratarem de serviços de urgência, devem, necessariamente, funcionar vinte e quatro horas por dia, sete dias por semana.

\section{COMENTÁRIOS}

Podemos concluir, ante ao exposto, que a estrutura de um CCI é complexa e dinâmica. Por esta razão, este tipo de entidade deve atuar em um vasto campo multidisciplinar e integrar-se às instituições sociais, de saúde, de ensino e pesquisa e à comunidade, a fim de cumprir com sua finalidade precípua, isto é, prevenir a ocorrência de intoxicações exógenas $1,4,9,10,12,13,15,16,17,18,19,20,22,23,24,25,26$, $28,29,30,32,34$. Evidencia-se, então, a necessidade de enfatizar a sua intervenção na área de promoção e proteção à saúde, ao invés de voltar sua atuação principalmente para a área de recupera- 
BAROUD, R. Concepção e organização de um centro de controle de intoxicações. Rev. Saúde públ., S. Paulo, 19:556-65, 1985.

ção da saúde ${ }^{15,20,23}$. Segundo este pensamento, torna-se fundamental o estabelecimento de algumas linhas de ação a nível educacional, de saúde mental, de capacitação profissional e de apoio técnico-científico, extrapolando o simples controle da incidência de determinadas síndromes tóxicas, a exemplo do que já foi feito em alguns centros internacionais. A seguir, serão abordados alguns aspectos destas linhas de ação de um modo geral e sucinto.

Deve-se à atuação a nível educacional a formação de uma comunidade ciente dos riscos toxicológicos a que está sujeita quando exposta a uma gama imensa de agentes potencial e efetivamente tóxicos. Através desta conscientização, é possível estabelecer meios mais seguros para a convivência e utilização destes agentes e, ainda, contar com uma maior possibilidade de participação da comunidade. Esta atuação apoia-se na informação dos riscos toxicológicos e na orientação sobre o uso e a manipulação adequados de produtos domissanitários e de uso industrial, praguicidas, drogas e outros, bem como na divulgação de cuidados de armazenagem (em locais apropriados e fora do alcance das crianças) e rotulagem (mantendo-os em sua embalagem original, sem reaproveitá-las) e de noções de primeiros-socorros. O caráter desta atuação pode ser abrangente, visando a comunidade como um todo através de divulgação pelos meios de comunicação $1,4,18,22,24,25,32$ e de eventos periódicos como, por exemplo, a Semana Nacional de Prevenção de Acidentes Toxicoló$\operatorname{gicos}^{1,4,13,15,17,20,26,29,30,32}$, Semana da Criança*, Feira do Verde** e outros. Esta atuação pode, também, adquirir um caráter mais restrito e específico como, por

- O CCI (SP) participou deste evento realizado na cidade de São Paulo, SP, durante os anos de 1976 a 80 e em 1982.

* O CCI (SP) participou deste evento, realizado na cidade de São Paulo, SP, durante os anos de 1976 a 80 . exemplo, dirigida a crianças e aos pais $\mathrm{e}$ educadores $4,15,16,25,26,29,34$.

Como foi mencionado anteriormente, através dos estudos epidemiológicos desenvolvidos pelos CCIs, e por outras entidades, são revelados os fatores etiológicos envolvidos nas intoxicações, assim como são detectados os aumentos das incidências de determinadas intoxicações $1,4,9,10,14,15,22,28,32$. Deste modo, é possível estabelecer ações que visem a controlar estas incidências. Pode-se citar, como exemplo, os programas de prevenção do saturnismo e de prescrição abusiva de medicamentos desenvolvidos nos EUA $^{26}$. Através destes estudos pode-se, ainda, adequar a regulamentação da produção, distribuição, e comercialização de substâncias biológica e farmacologicamente ativas. Estas adequações podem referir-se, por exemplo, (a) à obrigatoriedade do uso de embalagens de determinados produtos que dificultem o acesso das crianças ${ }^{4,11,15,26,29,30,33}$, (b) à indicação da formulação, riscos à saúde e primeiros-socorros de produtos industrializa$\operatorname{dos}^{4,14,13,17,22}$ e (c) à proibição da utilização de substâncias de comprovada toxicidade $^{14,15}$ e da veiculação publicitária destes produtos 4 .

As intoxicações auto-induzidas apresentam uma incidência crescente e muito relevante em nosso meio ${ }^{2,21}$ e por isso, merecem atenção especial. O estabelecimento de uma linha de ação a nível de saúde mental objetiva um atendimento integral aos pacientes que, por meio de uma intoxicação exógena, tentam suicídio. Este atendimento pode ser dividido em dois momentos, o momento crítico da internação $\mathrm{e}$, posteriormente, o acompanhamento destes pacientes. No primeiro momento é de fundamental importância que o paciente receba um adequado atendimento psicossocial para que se possa avaliar todas as causas envolvidas na intoxicação. Quando houver necessidade do prosseguimento deste atendimento, o 
BAROUD, R. Concepção e organização de um centro de controle de intoxicações. Rev. Saúde públ., S. Paulo, 19:556-65, 1985.

paciente será encaminhado aos recursos de saúde mental da comunidade (por exemplo, Ambulatórios de Saúde Mental, Comunidades Terapêuticas, Associação dos Alcoólatras Anônimos, Centros de Valorização da Vida, entre outros) ${ }^{19}$. Daí resulta a enorme importância dos CCIs integrarem-se a estes organismos, o que favoreceria efetivamente a reinserção destes pacientes em seu meio social contribuindo para evitar a reincidência.

A nível de capacitação profissional o estabelecimento de programas destinados aos profissionais e acadêmicos ${ }^{5,12,15,17,18,}$ 22,23 têm por objetivo contribuir para uma melhor formação científica na área de toxicologia de urgência. A transmissão de informações acerca da epidemiologia e da conduta terapêutica e analítica nas intoxicações, de programas de prevenção e controle de intoxicações, de noções de organização e atuação de um CCI e outros, seriam fornecidas através de cursos, palestras, simpósios, congressos e atividades acadêmicas.

Finalmente, um CCI pode desenvolver trabalhos de investigação científica sobre todos os aspectos envolvidos nas intoxicações exógenas $1,10,12,15,17,18,20,22,24,32$ em colaboração com instituições de ensino e pesquisa, uma vez que há uma relevante carência de dados disponíveis sobre os efeitos tóxicos de substâncias químicas no organismo de adultos, crianças, gestantes e recém-nascidos expostos, aguda e cronicamente. Este apoio técnicocientífico fornecido pelo CCI contribuirá efetivamente para o desenvolvimento destas investigações, proporcionando, indiretamente, condições mais adequadas de saúde à comunidade.

\section{CONCLUSOEES}

Para que os centros ligados às intoxicações exógenas existentes atualmente em nosso meio possam cumprir eficaz- mente com seus objetivos, e, como contribuição para o planejamento daqueles a serem implementados, sugere-se que:

- sejam incluídos cursos básicos de toxicologia e noções gerais sobre estes centros na formação de profissionais da área de saúde;

- seja propiciada a reciclagem e treinamento dos profissionais atuantes nestes centros;

- sejam inseridos programas de prevenção e controle de intoxicações na rede de saúde, abrangendo tanto à área de promoção e proteção como a de recuperação da saúde;

- seja fomentado um maior inter-relacionamento, quer entre os centros nacionais, quer destes com os internacionais, além de uma efetiva integração dos mesmos à área social, de saúde e de ensino e pesquisa e,

- seja viabilizado um maior incentivo aos setores de Epidemiologia e Prevenção, de Análises Toxicológicas e de Pesquisa Científica dos CCIs, através da formação e aproveitamento de recursos humanos especializados e de um maior investimento financeiro na infra-estrutura dos mesmos.

Estas providências concorrerão para o maior esclarecimento e, conseqüientemente, para a diminuição da incidência de intoxicações, revertendo em economia de recursos financeiros que poderiam ser úteis para o atendimento integral de pacientes intoxicados.

\section{AGRADECIMENTOS}

A Daisy de Brito Rezende, do Instituto de Química da Universidade de São Paulo e ao Prof. Fausto Antonio de Azevedo, do Curso de Farmácia da Pontifícia Universidade Católica de Campinas, pelas sugestões. 
BAROUD, R. Concepção e organização de um centro de controle de intoxicações. Rev. Saúde públ., S. Paulo, 19:556-65, 1985.

BAROUD, $R$. [The conception and organization of a center for the control of cases of poisoning]. Rev. Saúde públ., S. Paulo, 19:556-65, 1985.

ABSTRACT: As a contribution to a better understanding of the importance of Poison Control Centers, the present work focuses on their conception, organization and action as part of government institutions, private entities and the community. The importance of the sectors that deal with Prevention and Epidemiology, Toxicological Analysis and Scientific Research in these centers is particularly emphasized. Also the necessity for intervention in the areas of preventive public health by means of the creation of plans of action, particularly those related to public awareness, education and mental health, is stressed. Finally suggestions are made which, if implemented, should facilitate the understanding of cases of poisoning and hence diminish their incidence; this should result, in turn, in economy of available government resources, permiting development of more comprehensive treatment programs for patients suffering from poisoning.

UNITERMS: Poison Control Centers. Poisoning, prevention and control.

\section{REFERENCIAS BIBLIOGRĂFICAS}

1. ADAMS, W.C. Poison control centers: their purpose and operation. Clin. Pharmacol. Therap., 4: 293-6, 1963.

2. AMARAL, D.A.; BAROUD, R.; BRICKS, L.F.; MARTINS, L.A.; REIMÃO, R.N.A. \& SUGTYAMA, M. Intoxicações exógenas em adultos. [Apresentado ao III Congresso Brasileiro de Toxicologia e I Congresso Brasileiro de Acidentes e Intoxicação na Infância, Belo Horizonte, MG, 1983].

3. ASSOCIATION EUROPEENNE DES CENTRES DE LUTTE CONTRE LES POISONS. Les centres anti-poisons europeans et extra europeens. 8ème ed. Bruxelles, 1984.

4. BRAINI, A.D.; RUNTI, G. \& MARCHI, A.G. Consideraziono sugli avvelenamenti del bambino e sulla loro prevenzione. Min. Pediat., 34: 207-14, 1982.

5. COMSTOCK, E.G.; FERNANDEZ, G.; ESPONDA, A.C.; DECEDO, H. \& DE GARBINO, J.P. Toxicology and clinical toxicology in Uruguay. Clin. Toxicol., 18: 635-41, 1981.

6. CROTTY, J.J. \& VERHULST, H.I. Organization and delivery of poison information in the United States. Pediatr. Clin. N. Amer., 17: 741-6, 1970.

7. DECKER, W.J. Laboratory support of drug abuse control programs: an overview. Clin. Toxicol., 10: 23-35, 1977.
8. DECRETO n. 9.652, 27 set. 1971: Criação do Centro de Controle de Intoxicações da Prefeitura do Município de São Paulo. Diário Oficial do Município, São Paulo, 28 set. 1971.

9. EFTHYMIOU, M.-L.; VICENT, V. \& JOUGLARD, J. Les centres anti-poisons. Définition, fonctionnement et rôles. $J$. Eur. Toxicol., 6: 254-8, 1973.

10. GERVAIS, P. \& MOTIN, J. L'intégration des centres anti-poisons dans le centre hospitalo-universitaire. J. Eur. Toxicol., 2: $163-6,1969$.

11. GIOVACHINI, R.P. Toxicological evaluation of product safety. Pediat. Clin. N. Amer., 17: 645-52, 1970.

12. GOULDING, R. Poisons control centers. An essay on information and prevention. In: Hayes Jr., W.J. ed. Essays in toxicology. New York, Academic Press, 1975. p. $79-100$

13. GOVAERTS, M. Poison control in Europe. Pediat. Clin. N. Amer., 17: 72939,1970 .

14. GOVAERTS- LEPICARD, M. Epidemiology in chilhood poisoning: implication in prevention planning. Clin. Toxicol., 18: $1145-8,1981$.

15. GOVAERTS-LEPICARD, M. Papel dos centros de controle de intoxicações. Saúde ocup. segur., 19(1): 42-8, 1984. 
BAROUD, R. Concepção e organização de um centro de controle de intoxicações. Rev. Saúde públ., S. Paulo, 19:556-65, 1985.

16. KRENZELOK, E.P. \& GARBER, R.J. Teaching poison prevention to preschool children, their parents, and professional educators through child care centers. Amer. J. publ. Hlth, 71: 750-2, 1981

17. LOVEJOY Jr., F.H. \& ALPERT, J.J. A future direction for poison centers: a critique. Pediat. Clin. N. Amer., 17: 747-53, 1970.

18. LUCKENS, M.M. Poison central: an integrative approach to poison control. Amer. J. publ. Hlth, 58: 2290-4, 1968.

19. McCUlLOGH, D.K.; SANGSTER, G. \& LAWSON, A.A.H. Can recurrent selfpoisoning be prevent? A critical review of poisoning in West Fife, 1975-79. Practitioner, 225: 879-83, 1981.

20. MILLER, R.R, \& JOHNSON, S.R. Poison control. Now and in the future. Amer. J. Nurs., 66: 1984-7, 1966.

21. PAIVA, L.M. Tanitismo. Definições. In: Paiva, L.M. Depressäo e suicídia: psicanálise psicossomática, tanitismo. Rio de Janeiro, Imago, 1980. v. 2, p. 30-69; 202-11.

22. PICAZO-MICHEL, E. IV. Utilidad y objetivos de un centro de intoxicados. Gac. med. Mexico, 105: 369-75, 1973.

23. ROBB, G.L.; ELWOOD, H.S. \& HAGGERTY, R.J. Evaluation of a poison center. Amer. J. publ. Hlth, 53: 1751.60, 1963.

24. ROBERTSON, W.O. \& AMBUEL, J.P. Incorporation a poison control center into a pediatric "teaching" program. $J$ med. Educ., 37: 217-9, 1962.

25. RODRIGUES, Y.T. \& RODRIGUES, P. P.B. Intoxicação exógena e choque em pediatria. J. Pediat., 49: 391-4, 1980.

26. SCHERZ, R.G. Prevntion of childhood poisoning. A community project. Pediat. Clin. N. Amer., 17: 713-27, 1970
27. SCHERZ, R.G. \& ROBERTSON, W.O. The history of poison control centers in the United States. Clin. Toxicol., 12: $291-6,1978$

28. SCHVARTSMAN, S. Estrutura e objetivos de um centro de controle de intoxicações. Clin. Pediat., 3: 24-6, 1978.

29. SCHVARTSMAN, S. Profilaxia das intoxicações. In: Schvartsman, S. Intoxicações agudas. $2 .^{2}$ ed., São Paulo, Sarvier, 1979. v. 3, p. 83-8.

30. SCHVARTSMAN, S.; VAZ, F.A.C. \& SOBRINHO NETTO, A.H. Aspecto médico-sociais das intoxicações em crianças. Análise de 1.600 casos. Rev. Hosp. Clin. Fac. Med. S. Paulo, 27: $65-70,1972$.

31. SUNSHINE, I.; GOVAERTS, A.; GAULTIER, M.; ROCHE, L. \& VINCENT, V. Amerique. In: Sunshine, I, et al, Les centres anti-poisons dans le monde. $\mathrm{Pa}$ ris, Masson, 1966. p. 48-58.

32. SUNSHINE, I.; GOVAERTS, A.; GAULTIER, M.; ROCHE L. \& VINCENT, $V$. Conception et organization des centres de poisons. In: Sunshine, I, et al. Les centres anti-poisons dans le monde. Paris, Masson, 1966. p. 201-14.

33. TEMPLE, A.R. Testing of child-resistant containers. Clin. Toxicol., 12: 357-65, 1978.

34. TRUDEAU, T.W. \& BRADEN, F.M. The development of a unique, pharmacy based, poison prevention program. Hosp. Top., 56: 32-6, 1978.

Recebido para publicação em 22/03/1985

Reapresentado em $04 / 09 / 1985$

A provado para publicação em 10/09/1985 